Pak. j. sci. ind. res. Ser. B: biol. sci. 2018 61B(3) 172-181

\title{
Review
}

\section{Epigenetic Alterations and their Dietary Backgrounds}

\author{
Rani Saira Saleem ${ }^{a *}$ and Rani Wafa Shear ${ }^{b}$ \\ ${ }^{a}$ Institute of Biochemistry and Biotechnology, University of Punjab, Lahore, Pakistan \\ ${ }^{b}$ Biotechnology Department, University of Gujrat, Pakistan
}

(received June 27, 2016; revised April 25, 2017; accepted May 10, 2017)

\begin{abstract}
The key intention of this review is to summarize the different studies which relate the genomediet interactions with future perspectives of exploring an insight into the well defined functions of diverse micronutrients and other dietary components that play a vital task in defining the early developmental patterns of an organism. Human fetus development is a complex process that is totally dependent on the dietary components which interact with the genes to regulate the different proliferation and differentiation stages. We want to explore those complex interactions that lay hidden between micro-nutrients and gene expressions but are means of the apparent changes of a phenotype of an individual. Along with this the review will also perceive some basics of development of certain diseases as well, due to these complex genome-diet interactions thus leading to refine the dietary outlines for maternal and prenatal developmental stages in future. Research has also shown that genome-diet interactions are very complex as without proper nutrients the end result is the genome instability which may lead to chronic diseases, developmental defects and certain types of cancer.
\end{abstract}

Keywords: epigenetics, genome diet, methylation, DNA sequence, eukaryotes

\section{Introduction}

The definition of the term 'Epigenetics' was given by Waddington in 1942 while, discussing the modulation of development by gene regulation. He presented the basic idea for the interaction of genes with their environment thus concluding a particular phenotype (Waddington, 1953). Epigenetics is the branch of biology which deals with the study of heritable cellular and physiological traits, which are not characterized by alterations in the DNA sequence. In the most widely studied epigenetic changes include the DNA structural changes during the cell differentiation process or morphogenesis at early developmental stages. They may include methylation of DNA or modifications of histones which might be responsible for changing the expression of certain genes playing their role in the right development of a life form. Epigenetic modifications actually translate the relationship between heredity and environment and amongst the environmental factors the dietary exposure serves as the most important clue. Experimental procedures and statistical data obtained from different studies relate that the maternal and fetus dietary exposure in the first 1000 days of life is very important. This is also called as 'primary dietary

*Author for correspondence;

E-mail: ranisairasaleem@hotmail.com exposure'. This primary dietary exposure has been found responsible for bringing up and carrying the epigenetic changes that might last for the whole life of an individual or might affect the individual's generation after generation by regulating chromatin remodeling. The early micronutrient status of fetus holds enough strength to alter the metabolism rate, organ growth, and regulate the genes responsible for cell differentiation and organogenesis process. It refers to such external modifications responsible for turning the genes on and off which are other than involving the actual DNA sequence (Waddington, 1953).

In this case the actual coding sequence of DNA is not affected but DNA structure itself gets modulated in such a way that certain genes are turned on or off as a result. Interestingly nowadays, Epigenetic marks are recognized as mechanistic links between environment, nutrition and genes. DNA modifications or epigenetic alterations serve as the basis of changes in the metabolism rate, cell differentiation pattern, brain development and organogenesis of the fetus by manipulating the expression of developmental genes and a network of transcriptional factors. These epigenetic changes are under the strong influence of environmental interactions which affect the developmental stages in one way or the other. With the increasing understanding about the 
field of epigenetics, it becomes clearer that epigenetic incidents or inherited changes in the gene expression that take place without any alteration in the genomic DNA sequence are important thus leading towards cancer. The epigenetic mechanisms actually decide the fate of the cell and also build up certain barriers to prevent the reversion to preceding cellular states (Reik, 2007). The development of a cell starts from a pluripotent stage from which it changes into many cell types and then to tissues and organs, respectively. The factors which direct the translation of this pluripotent staged cell to such a complex form also include the dietary factors or various necessary micronutrients like iron, calcium, vitamin A \& D, zinc and magnesium etc. The gene expression programme becomes more effective, well established and potentially efficient in the right direction, well driven by the force of these micronutrients which are needed in small amounts but are indispensible for the development of fetus by all means.

The study of micro-nutrients and their pathways in the body may lead us to a clear understanding that they might play a significant role by interacting with the epigenetic mechanisms. The chromatin modifications which are resulted as environmental response can be reversed, are temporary and allow adaptations. In some situations these might keep on moving throughout the existence of an individual. This is also called as metabolic imprinting which is regarded as the result of motherly nourishment that is given during development and the suckling period on DNA patterns and gene regulation patterns that operate lifetime risk of chronic diseases (Waterland and Garza, 1999).

Chromatin conformation and regulation of epigenetic changes. The packaging of huge amount of DNA into their nucleus is a tedious task which a eukaryotic cell has to go through. The eukaryotic DNA consists of transcriptionally active DNA called Euchromatin and transcriptionaly incompetent DNA that is Heterochromatin. As result of this complex packaging process a basic unit nucleosome of about 146bp comes into play which is laterally epigenetically modified under the effect of various factors to allow the cell to control transcriptional activity and genetic expression (Rountree et al., 2001). The organization of eukaryotic chromatin is still partially understood. The technique like X-ray crystallography has enabled us to have a deep insight into the detailed structure of the essential repeating element of chromatin that is nucleosome.
There are more than about 30,000 genes in our genome functional in various body parts of an organism. This varied expression of genes depends on variety of transcription factors. Scientists have shown via in vitro and in vivo experiments that although cells of body are identical yet they are different in their expression profiles. The remarkable chromatin modifications that are well studied in eukaryotes include methylation of DNA patterns, modifications taking place in histones and functioning of small RNAs both considerately and independently to constitute the epigenome which transmit gene expression outline via the process of mitosis/meiosis (Surani, 2013). Such epigenetic modifications together have emerged as critically important for the regulation roles in the transcriptional rheostat.

DNA methylation. It is the characteristic variation in eukaryotic DNA which involves methylation of cytosines at the carbon 5 position of the $\mathrm{CpG}$ dinucleotides. $\mathrm{CpG}$ sites are the regions of DNA in which cytosine nucleotide is followed by Guanine. $\mathrm{CpG}$ is shorthand for 5 ' $-\mathrm{C}-$ phosphate $-\mathrm{G}-3$ '. These regions often serve as promoters or transcription start sites. Cytosines in $\mathrm{CpG}$ dinucleotides can be methylated to form 5methylcytosine (Jabbari and Bernardi, 2004). 5 methylcytosine performs similar functions as that of normal cytosine. Methylation of DNA can also be continued directly from the germ line silencing any one of the parental chromosome also known as genomic imprinting. The eukaryotic genome can be categorized into two groups, with methylated and non methylated DNA. In mammals about $70 \%$ methylated $\mathrm{CpG}$ 's content and only 1-2\% DNA consists of non methylated CpG.

The initial information and identification of the methylated DNA was stated by experiments performed by Razin and Cedar, 1977 (Ball et al., 1983). It was almost after a decade that micro-injection experimentation confirmed that DNA methylation via vivo and in vitro methylated DNA sequences exposed that it results to inactivate the chromatin material (Keshet et al., 1986). The genomic methylation patterns in mammals that take place during embryogenesis involve overall three DNA methyltranferases DNMT1, DNMT3a, DNMT3b. These are also known as de novo methyltransferases which are meant to ascertain the methylation outline during developmental stages (Okano et al., 1999). Later on the identification of two methyl binding proteins $\mathrm{MeCP} 1$ and $\mathrm{MeCP} 2$ assisted to understand that DNA methylation has its role to play 
with DNA structure and gene expression (Lewis et al., 1992; Meehan et al., 1989). Thus, concluding that DNA methylation is accountable for the transcriptionally inactive DNA. Any change in the methylation patterns at any stage of development of an organism may lead to abnormalities. Imprinting and methylation patterns in germ cells are shown in Fig. 1a (adapted from Rountree et al., 2001). The primary methyl donating specie for this process is SAM (S-Adenosyl metheonine) that is regarded as a molecule of cyclic cellular process called as one carbon metabolism.

This methylation of DNA is liable for the regulation of thousands of genes present in the genome of an individual. Methylation is involved in the processes of:

- Inactivation of an X-chromosomes in females

- Genetically imprinted genes

- Silencing of certain genes

Histone modifications. At molecular level specific chromatin domains can be recognized by the help of histone modifications. The different type of histone modifications include methylation, phosphorylation, acetylation, sumoylation and ubiqutination of histones residues. Such different changes in histones might affect the transcriptional patterns of genomic DNA (Schneider et al., 2004; Offield et al., 1996). The methylation of histone which takes place at specific residues like lysine and arginine straightly influences the action and functions of DBP (DNA binding proteins) thereby regulating the expression of genes and thus affecting the strength of the genome (Mosammaparast and Shi, 2010). The acetylation of histones is one of the main frequent transcriptional controls adapted by most of the eukaryotes

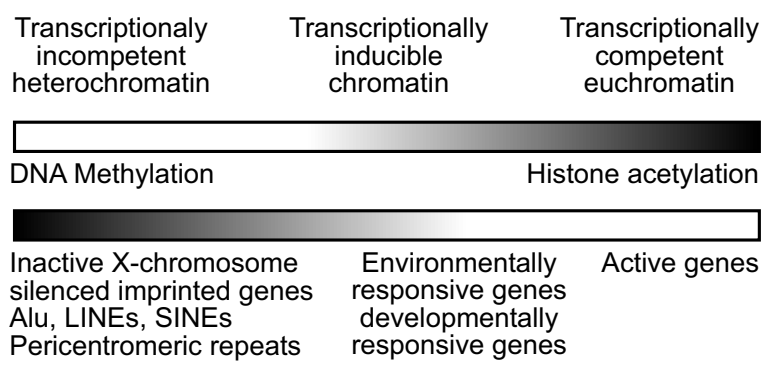

Fig. 1a. The transcriptional rheostat. DNA methylation and histone acetylation help to establish chromatin states that either foster or inhibit transcription (adapted from Rountree et al., 2001).
(Grunstein, 1997). The mechanism involved in this control includes acetylation of $\mathrm{NH}_{2}$ terminal of $\mathrm{H} 3$ and $\mathrm{H} 4$ by histone acetyltransferases (HATs) which will facilitate the transcriptional activity of the genes. However, this transcriptional activity is stopped by removing the acetyl group by histone deacetylases (HDACs). The HATs and HDACs are used as the co activators and repressors of the transcriptional machinery of the cell, respectively (Rountree et al., 2001).

There have been several different proteins that have been identified for their roles in the transcription repression (MBD1, MBD2, MBD3, and MeCP2) (Wade, 2001). They are supposed to act for the repression of transcription during the function of HDACs. This relationship was indicated earliest for $\mathrm{MeCP} 2$ protein (Nan et al., 1998; Jones et al., 1998). MeCP2 has also its role in anchoring a multiprotein repressory complex to the DNA which is responsible for deacetylation of histones and alterations of chromatin by recruiting deactylases HDAC1 and HDAC2 also shown in Fig. 1b. Experiments were performed to show that transcriptional inactivation which is sourced by the deacetylation might be lessened by HDAC inhibitor Trichostatin A (TSA) (Eden et al., 1998; Jones et al., 1998; Nan et al., 1998). Such explanations and studies resolve the supposed pathway of gene silencing by relating finally the long three method suspected correlation between DNA methylation, chromatin remodeling and expression of genes (Razin, 1998).

One carbon metabolism pathway and SAM. Both the above types of DNA modifications are interlinked by their common dependence on S-Adenosyl Methionine

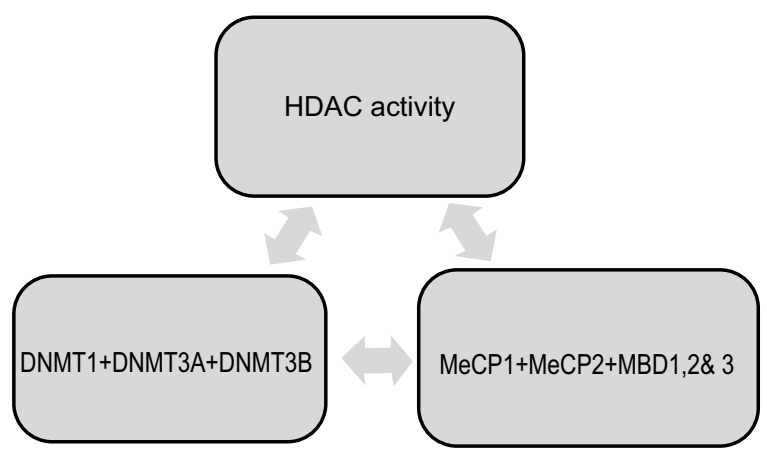

Fig. 1b. The interrelationship between DNA methylation and Histones modification Transcriptional Regulatory Machinery. 
which plays central role as methyl donor. It is important to remember here that almost all the DNA and histone methyltranferases require this cofactor to transfer methyl group to cytosine, lysine or arginine residues. This is an important co enzyme that carries the transmethylation reactions that takes place in liver. SAMC (S-Adenosylmetheonine Mitochondrial Carrier protein) belongs to solute carrier family 25 members 26 , which consists of 274 amino acid proteins that transports SAM, as well as metabolites, nucleotides and cofactors, through the mitochondrial inner membrane. The one carbon metabolism involves TP driven reactions in which methionine is converted into S-adenosylmetheonine, a universal methyl donor. DNMTs help to put together methyl group from SAM to carbon 5 position of cytosine therefore, forming 5-methyl cytosine and resulting in methylation.

S-adenosylmetheonine was for the first time discovered by Giulio Cantoni also known as AdoMet. It is conjugate of methionine and ATP. The formation of SAM is catalyzed by MAT i.e. Methyl Adenosyltransferase also known as SAM Synthase. Regeneration of SAM can take place by the demethylation of SAM via the methionine cycle taking place in liver. This methionine cycle also called one carbon metabolism is folate dependent as shown in Fig. 2. Any irregulation of this methionine cycle is the result of the folate deficiency via dietary insufficiency or lack of balance diet which may result in dictating various hereditary changes (Loenen, 2006). One other important function that has been demonstrated for SAM is that it also serves as cofactor for the activity of nucleases EcoK1 which can not otherwise restrict DNA in the absence of SAM. MTR enzyme of methionine synthase is an essential enzyme for catalyzing the 5-methyl THF induced remethylation of Homocysteine to Methionine (Stover, 2011). This MTR is a cobalamine (Vit B-12) reliant enzyme which performs its function well provided the presence of sufficient amount of this vitamin in the cell.

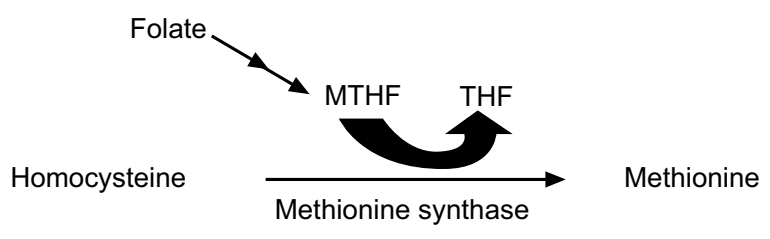

Fig. 2. Folate mediated induction of $1 \mathrm{C}$ metabolism.
One carbon metabolism is a mechanism that is present in the mitochondria, cytoplasm and nucleus as well and this pathway is induced by the presence of dietary micronutrients as cofactors, like folate and also known as folate mediated 1-C metabolism. This pathway has been studied for the de novo synthesis of purines, thymidylate and methionine. This is because these entire pathways involve Tetrahydrofolate (THF) as cofactor which is the precursor of folate (Stover and Fox, 2008).

In one carbon metabolism mitochondria utilizes the cofactor THF to generate formate from the amino acids serine, glycine, and from sarcosine and dimethylglycine which are the result of choline degradation. The formate is then transported to cytoplasm where its condensation with THF in an ATP dependent environment takes place and 10 Formyl THF is resulted (Caudill and Stover, 2008). The formate generation is critical for the AdoMetdependent methylation reactions, including chromatin methylation and also it serves as the primary source for the de novo biosynthesis of purines and for homocysteine remethylation to methionine (Appling, 1991). It is important to consider here that a mammalian cell has many different AdoMet dependent methyltransferases which are involved in different cell processes like chromatin remodeling, regulatory functions for gene transcription (Miranda and Jones, 2007), protein compartmentalization (Winter-Vann et al., 2003), and the biosynthesis and catabolism of small molecules including neurotransmitters. S adenosylehomocysteine is the product that is resulted from the AdoMet depenedent transmethylation reactions.

One carbon metabolism is highly sensitive to the dietary components i.e. vitamins (Herbig, 2002). Certain dietary components are significant to show genomic interactions and folate is one of them as shown in Fig. 3 adapted from Jing (1997). A follow up study by health professionals indicated that the use of alcohol significantly overcome the effects of genetic mutations responsible for colorectal cancer because ethanol has the cleavage ability for folate and it also inhibits the absorption and utilization of folate and increases its excretion. Furthermore, alcohol acts like a methyl group antagonist thereby, causing imbalanced DNA methylation (Jing, 1997).

The purines and thymidylate are required for DNA synthesis whereas; SAM is required as a part of DNA methylation factory. This folate dependent synthesis of DNA and SAM precursors for genome programming 


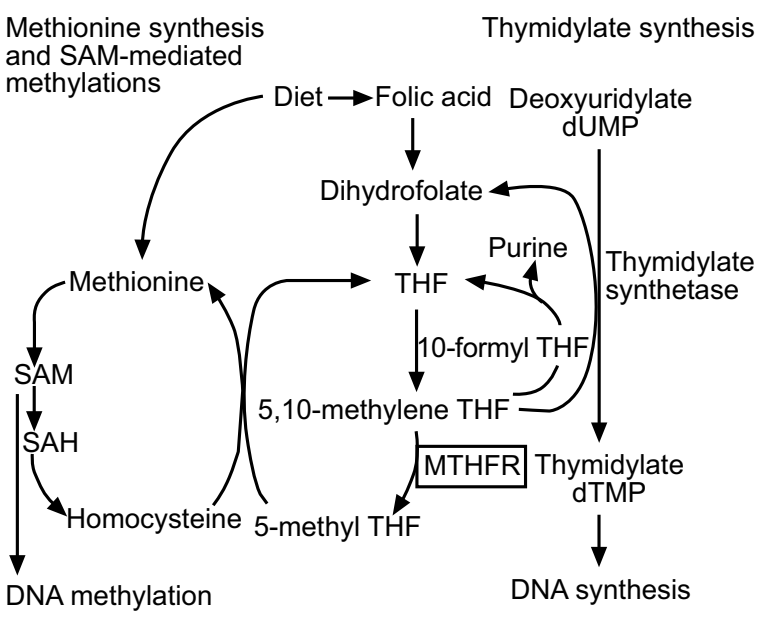

Fig. 3. An overview of one carbon metabolism and dietary factor involved (Adapted from Jing Ma, 1997).

is largely dependent on the availability of many vitamins and minerals at the same time. Thus we can say that the folate dependent one carbon metabolism serves as a central relationship between nutrient status and genomic programming during the fetal development and this nutritional pool is developed by the maternal dietary background (Stover, 2007). Any sort of disturbances in this folate mediated 1-C metabolism increases the risk of certain cancers, cardiovascular diseases, neurological disorders and developmental anomalies such as spina bifida, cleft palate and spontaneous abortions (Stover, 2004). However, the folate supplementation reduces the risks of diseases and developmental disorders in the population (DeMarco, 2000).

The role of micronutrients in DNA modifications. The intake of micronutrients and balance diets offers the biggest challenge in regulation of the metabolic and differentiation processes taking place at the time of development of fetus. As the growing fetus is largely dependent on the micronutrient status of the mother therefore, pregnant woman is at high risk of micronutrient deficiencies (Doyle et al., 1992). For understanding the interaction between nutrients and epigenetic mechanisms one need to have a complete insight into the physiological roles of micronutrients for maternal and prenatal health. The pregnancy outcomes can be affected by the micronutrients by resulting alterations in maternal and fetal metabolism by changing their role, enzyme activities, signal transduction and transcription pathways (Berti et al., 2011). Micronutrients distort the metabolism and genetic regulations in a number of complicated pathways. The folate dependent biosynthesis of chromatin material for DNA synthesis and methylation is dependent on the supply of many vitamins including B-12, B-6, niacin, riboflavin and minerals which are actually responsible for DNA synthesis and modifications.

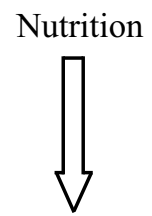

Physical appearances

The embryonic stages are the most sensitive stages that immediately respond to the nutrient induced adaptations in the gene expression. This phenomenon is labeled as metabolic imprinting or metabolic programming (Waterland and Garza, 1999). However these adaptations come into play within the early critical windows of development and lasts for adulthood. The above explained relationship between maternal nutrients status and fetal epigenetic programming actually serves as the basis for fetal origin of adult disease hypothesis which proposes that nutrition acts very early in life to predict the risks for adverse outcomes of the coming adult life (Barker, 1997). On the other hand genetic variations in the epigenetic programming can also impair the nutrition absorption and utilization which may result in the differences in the nutrient tolerances such as iron tolerance and thus lead to the variations in the nutrient requirements of the body. Iron deficiency is responsible for causing the reduced motor skill development of the growing children along with reduced power of learning and memorization (Carter et al., 2010).

The global methylation effects of maternal folate status have been followed in different studies till now. One of them clearly indicates that there was a considerable decrease in the small intestinal tissue as adults when an offspring was exposed to low folate dietary intake during the gestation and lactation period (McKay et al., 2011). Another study also related that the intake of folate according to the recommended dose in US women of child bearing age reduced the global methylation levels in murine colorectal tissue which was calculated by the liquid chromatography mass spectrometry (Sie et al., 2011). A number of studies have been done for studying the impacts of global methylation patterns on the colorectal cancer in post menopause women. These 
studies actually analyzed the global DNA methylation patterns by the indirect incorporation of ${ }^{3} \mathrm{H}$ methyl SAM (Pufulete et al., 2005a). The patients which were diagnosed with colorectal cancer when given an increased folate supplementation they showed an increase in the methylation patterns thus showing a direct correlation between folate supplementation and colorectal cancer prognosis (Pufulete et al., 2005b).

Gene specific studies have enabled the researchers to explore the impact of folate mediated DNA methylation patterns on various genes responsible for anomalies like cancer and diabetes. This has enabled us to explore the window for designing the therapeutics and preventive strategies from these anomalies by the help of dietary supplementation and hence targeting the epigenetic mechanisms. Till now the methylation patterns of different tumor related genes like NAT1, p53, several loci of CRC, and PPAR- $\alpha$, an insulin receptor gene have been related with the high or low folate mediated methylation pattern changes thus resulting in wide variety of expression changes (McKay et al., 2011; Wakefield et al., 2010; Burdge et al., 2009).

In addition to folate certain other micro nutrients have also been analyzed for their dietary influence on the methylation patterns. Choline is one of them which also serves as an indirect methyl donor in one carbon metabolism pathway. The effect of maternal choline deficient diet has been studied on three candidate genes cdkn3, cdkn2b and calb2. It was concluded that cdkn3 gene of mouse fetal brain of embryonic stage day 17 displayed a state of hypomethylation for its promoter region as exposed to choline deficient diet which proved the role of choline in the methylation mechanisms (Niculescu et al., 2006). Studies have also shown that early deficiency of choline in diet may cause the malfunctioning of methylation machinery.

The role of vitamin A has been studied in detail and it has been shown that vitamin $\mathrm{A}$ is critical for the regulation of alveolorization and septation in the lung development (Massaro and Massaro, 2010; 2006). This may relate to the hidden cause of some genes might get switch off and resulted in malformation of lung and its functions. However, the excess of vitamin A has also been related with the teratogenicity in humans (Biesalski 1989). Two studies conducted by Barreto et al. (1994) and Pearson et al. (1992) on the premature babies showed that the chances of chronic lung prematurity can be reduced to a larger extent by the administration of vitamin A. It is hypothesized that some genetic and metabolic pathways are correlated via this critical vitamin which hamper the development of the lungs in the premature babies (Biesalski, 2003). Vitamin A is converted to all trans retinoic acid (RA) which is transported to nucleus where it is interacted as an activating agent to the RA nuclear receptors. The induction of differentiation with retinoic acid and vitamin D3 was successfully conducted in myelodysplastic syndromes (Blaszek et al., 1990). The role of retinoic acid in preventing the tumor progression in central nervous system has been studies but the mechanism has still to be elucidated (Ross and Stephensen, 1996).

It is therefore, suggested that it may be the result of some regulatory role of certain transcriptional regulators or repressors to the thyroid hormone receptors. Vitamin A deficiency produces a number of malformation and differentiation abnormalities in the growing embryo. Figure 4 indicates all relationships that can be predicted among one carbon metabolic pathway and associated epigenetic regulations with the fetal nutritional status.

This review article illustrates the severe consequences and genetic diseases that are resulted from improper genetic programming and imprinting at the early developmental stages of the fetus. Above all, it focuses that the genetic diseases can be reduced to a larger extent by managing and monitoring the dietary needs of the fetus and maternal environment. Studies adapted from human epidemiological contexts and experimentation of the animal models clearly depicts that maternal nutrients can programme the gene expression and patterns of embryonic methylation that persist throughout the adult life and may contribute to many diseases (McMillen and Robinson, 2005). The importance of DNA methylation is clearly reflected by the study of large number of genetic diseases including cancer and summarized in Fig. 5.

The dietary vitamins and minerals continue to play an important role in lateral life and help in the management of cardiovascular, renal, and pulmonary functions. Every individual vitamin and mineral has its own characteristic role in managing a certain metabolic pathway for example calcium intake may help to keep an eye on blood pressure in adults (Van Mierlo et al., 2006). Zinc and magnesium have their well defined roles in insulin pathways and zinc metabolism has been studied in type 2 diabetes mellitus (Taylor, 2005; Chausmer, 1998; 
Garland, 1992). Therefore, it is concluded that the development of micronutrient deficiencies is although a slow process but may lead to severe consequences at the later stages of life as shown in Fig. 6 adapted from Shenkin (2004). The folate dependent 1-Carbon metabolism serves as a channel that links the cellular metabolism mechanisms to the epigenetic machinery of the cell. The common molecule that translates the information in this relationship is AdoMet. The cellular methylation patterns can be read AdoMet/AdoHcy ratio. The folate induced changes in the cell can influence the activity of DNA and histone methyl transferases thus controlling the chromatin methyl patterns. Yet it is still to elucidate the respective effects of these dietary dependent methlaytion patterns of different developmental genes like Hox clusters, shh signaling and FG

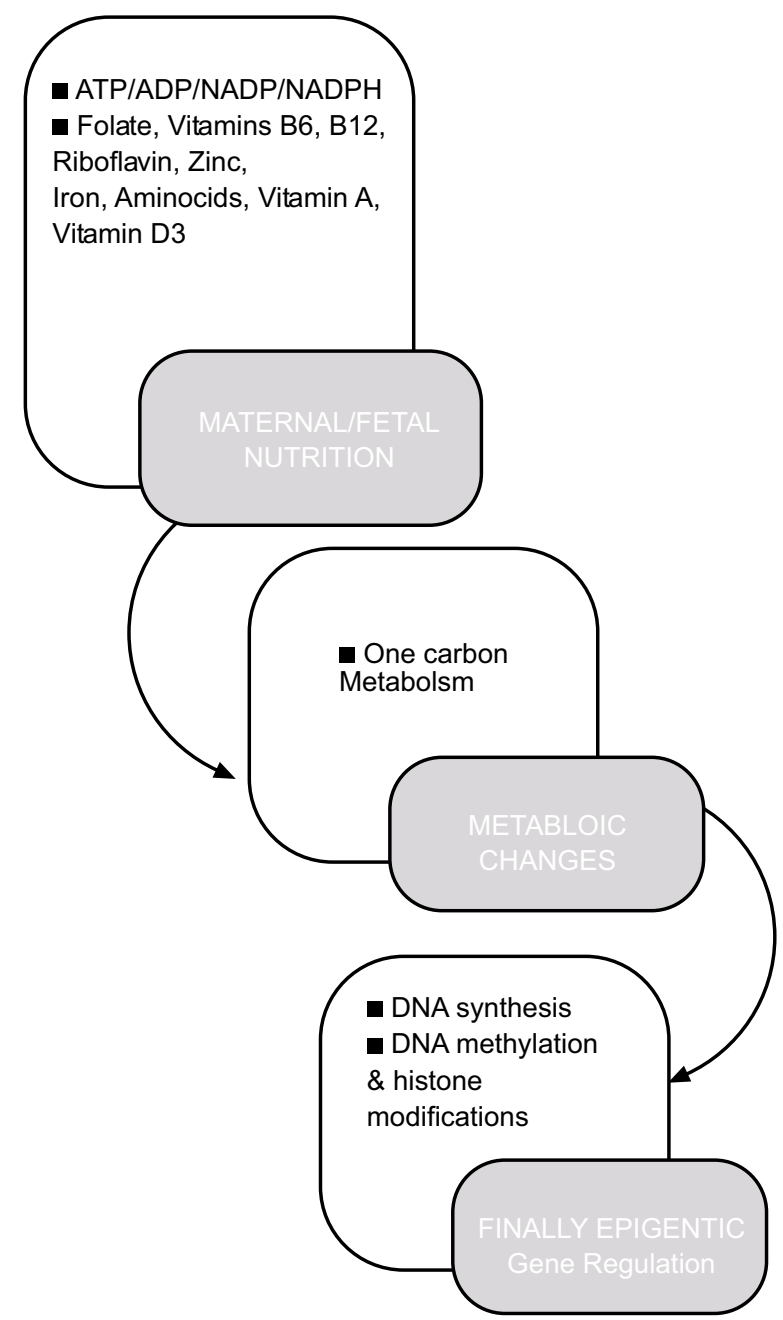

Fig. 4. Relationship between maternal/fetus nutrition and epigenetic changes. factors. The future research on these gene clusters, their transcriptional factors and domains, their protein products might give us a detailed insight of the type of the effects produced by these folate dependent mechanisms on development of the fetus. Furthermore, it is also important to emphasize on exploring the roles of other associated minerals on the one carbon metabolic pathways and on the methylation patterns. There is a need to explore the methylating patterns of individual genomic loci of some above mentioned candidate genes for developmental anomalies and then to study the regulating mechanisms of these candidate genes by following dose dependent micronutrients exposures. This will surely help us to elucidate the developmental roles played by these genes as well as their expression dependency on dietary components.

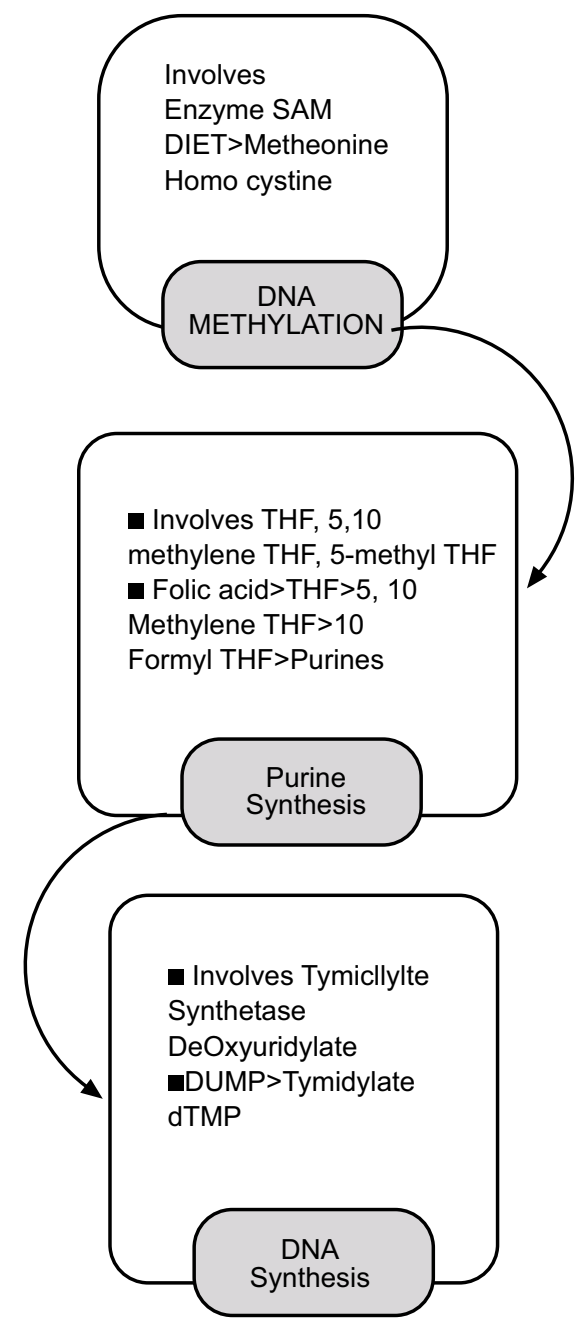

Fig. 5. Important cellular metabolic pathways and their relationship. 


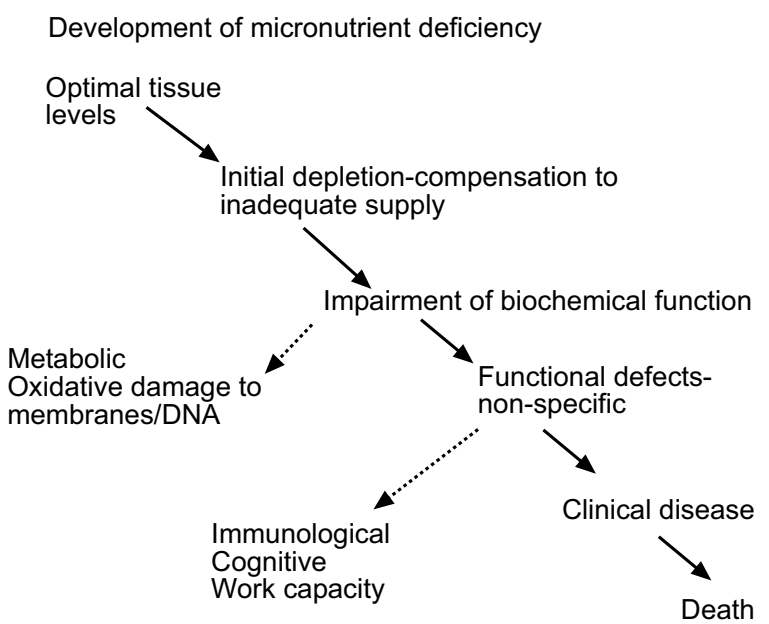

Fig. 6. Pathways for micronutrient deficiencies (Adapted from Shenkin, 2004).

\section{References}

Appling, D.R. 1991. Compartmentation of folatemediated one-carbon metabolism in eukaryotes. FASEB Journal, 5: 2645-2651.

Ball, D.J., Gross, D.S., Garrard, W.T. 1983. Proceedings of National Academy of Sciences, USA, 80: 54905494.

Barker, D.J. 1997. Intrauterine programming of Coronary heart disease and stroke. Acta Paediatrica Supplementum, 423: 178-182.

Barreto, M.I., Santos, I.M.P., Assis, A.M.O. 1994. Effect of vitamin A supplementation on diarrhoea and acute lower respiratory-tract infections in young children in Brazil. Lancet, 344: 228-231.

Berti, C., Biesalski, H.K., Bartner, R., Lapillone, A., Pietizik, K., Poston, L., Redman, C., Koletzko, B., Cetin, I. 2011. Micronutrients in pregnancy: Current knowledge and unresolved questions. Clinical Nutrition, 30: 689-701.

Biesalski, H.K. 2003. The significance of Vitamin A for the development and function of lung. Forum Nutrition, 56: 37-40.

Biesalski, H.K. 1989. Comparative assessment of toxicology of Vitamin A and Retinoid in man. Toxicology, 57: 117-161.

Blaszek, I., Mathè, G. 1990. Pathobiology of myelodysplastic syndromes. Biomedicine and Pharmacotherapy, 44: 69-83.

Burdge, G., Lillycrop, K.A., Phillips, E.S., SlaterJefferies, J.L., Jackson, A.A., Hanson, M.A. 2009. Follic acid supplementation during Juvenile- pubertal period in rats modifies the phenotype and epigenotype induced by prenatal nutrition. The Journal of Nutrition, 139: 1054-1060.

Carter, R.C., Jacobson, J.L., Burden, M.J., ArmonySivan, R., Dodge, N.C., Angelilli, M.L. 2010. Iron deficiency Anemia and cognitive function in infacncy. Pediatrics, 126: 427-432.

Caudill, S. 2008. Genetic and epigenetic contributions to human nutrition and health: managing genomediet interactions. Journal of American Diet Association, 108: 1480-1487.

Chausmer, A.B. 1998. Zinc, insulin and diabetes. Journal of American College of Nutrition, 17: 109-115.

DeMarco, P.M.A. 2000. Folate pathway gene alterations in patients with neural tube defects. American Journal of Medical Genetics, 95: 216-223.

Doyle, W., Wynn, A.H.A., Crawford, M.A. 1992. Nutritional counseling and supplementation in the second and third trimester of pregnancy, a study in a London population. Journal of Nutrition and Medicine, 3: 249-256.

Eden, S., Hashimshony, T., Keshet, I., Cedar, H., Thorne, A.W. 1998. DNA methylation models histone acetylation. Nature, 394: 842-843.

Garland, H.O. 1992. New experimental data on the reltionship between diabetes mellitus and magnesium. Magnesium Research, 5: 193-202.

Grunstein, M. 1997. Histone acetylation in chromatin structure and transcription. Nature, 389: 349-352.

Herbig, K.C.E. 2002. Cytoplasmic serine hydroxymethyltransferase mediates competition between folate-dependent deoxyribonucleotide and Sadenosylmethionine biosyntheses. Journal of Biological Chemistry, 277: 38381-38389.

Jabbari, K., Bernardi, G. 2004. Cytosine methylation and $\mathrm{CpG}, \mathrm{TpG}(\mathrm{CpA})$ and $\mathrm{TpA}$ frequencies. Gene, 333: 143-149.

Jing, M.A. 1997. Methylenetetrahydrofolate Reductase Polymorphism, Dietary Interactions, and Risk of Colorectal Cancer1. Cancer Research, 57: 10981102.

Jones, P.L., Veenstra, G.J.C., Wade, P.A., Vermaak, D., Kass, S.U., Landsberg, N., Strouboulis, J., Wolffe, A.P. 1998. Methylated DNA and MeCP2 recruit histone deacetylase to repress transcription. Nature Genetics, 19: 187-191.

Keshet, I., Lieman-Hurwitz, J., Cedar, H. 1986. DNA methylation affects the formation of active chromatin. Cell, 44: 535-543. 
Lewis, J.D., Meehan, R.R., Henzel, W.J., Maurer-Fogy, I., Jeppesen, P., Klein, F., Bird, A.P. 1992. Purification, sequence and cellular localisation of a novel chromosomal protein that binds to methylated DNA. Cell, 69: 905-914.

Loenen, W. 2006. S-Adenosylmethionine: jack of all trades and master of everything. Biochemical Society Transactions, 34: 330-333.

Massaro, D., Massaro, G.D. 2010. Lung development, lung functions and retinoids. New England Journal of Medicine, 362: 1829-1831.

Massaro, D., Massaro, G.D. 2006. Towards therapeutic pulmonary alveloar regeneration in humans. Proceedings of American Thoracic Society, 3: 709712.

McKay, J.A., Waltham, K.J., Williams, E.A., Mathers, J.C. 2011. Folate depletion during pregnancy and lactation reduces genomic DNA methylation in murine adult offspring. Genes and Nutrition, 6 : 189-196.

McMillen, I.C., Robinson, J. 2005. Developmental origins of the metabolic syndrome, prediction, plasticity and programming. Physiological Reviews, 61: 571-633.

Meehan, R.R., Lewis, J.D., McKay, S., Kleiner, E.L., Bird, A.P. 1989. Identification of a mammalian protein that binds specifically to DNA that contains methylated CpGs. Cell, 58: 499-507.

Miranda, T.B., Jones, P.A. 2007. DNA methylation: the nuts and bolts of repression. Journal of Cell Physiology, 213: 384-390.

Mosammaparast, N., Shi, Y. 2010. Reversal of histone methylation: biochemical and molecular mechanisms of histone demethylases. Annual Review of Biochemistry, 79: 155-179.

Nan, X., Huck-Hui, N.G., Colin, A., Johnson, Carol, D.L., Bryan, M.T., Robert, N.E., Adrian, B. 1998. Transcriptional repression by the methyl-CpGbinding protein $\mathrm{MeCP} 2$ involves a histone deacetylase complex. Nature, 393: 386-389.

Niculescu, M.D., Crciunescu, C.N., Zeisel, S.H. 2006. Dietary choline deficiency alters global and gene specific DNA methylation in developing hippocampus of mouse fetal brains. FASEB Journal, 20: 43-49.

Offield, M.J., Jetton, T.L., Labosky, P.A., Ray, M., Stein, R.W., Margnuson, M.A., Hogan, B.L., Wright, C.V. 1996. PDX-I is required for pancreatic outgrowth and differentiation of rostral duodenum. Development, 122: 983-995.
Okano, M., Bell, D.W., Haber, D.A., Li, E. 1999. DNA methyltransferases Dnmt3a and Dnmt3b are essential for de novo methylation and mammalian development. Cell, 99: 247-257.

Pearson, E., Bose, C., Snidow, T. 1992. Trial of vitamin A supplementation in very low birth weight infants at risk for bronchopulmonary dysplasia. Journal of Pediatrics, 121: 420-427.

Pufulete, M., Al-Ghnaniem, R., Khushal, A., Appleby, P., Harris, N., Gout, S., Emery, P.W., Sanders, T.A. 2005a. Effect of Folic acid supplementation on genomic DNA methylation in patients with colorectal adenoma. Gut, 54: 648-653.

Pufulete, M., Al-Ghnaniem, R., Rennie, J.A., Appleby, P., Harris, N., Gout, S., Emery, P.W., Sanders, T.A. 2005b. Influence of folate status on genomic DNA methylation in colonic mucosa of subjects without colorectal adenoma or Cancer. British Journal of Cancer, 92: 838-842.

Razin, A. 1998. CpG methylation, chromatin structure and gene silencing - a three-way connection. $E M B O$ Journal, 17: 4905-4908.

Razin, A., Cedar, H. 1977. Distribution of 5-methylcytosine in chromatin. Proceedings of the National Academy of Sciences, 74: 2725-2728.

Reik, W. 2007. Stability and flexibility of epigenetic gene regulation in mammalian development. Nature, 447: 425-432.

Ross, A.C., Stephensen, C.B. 1996. Vitamin A and Retinoids in antiviral responses. FASEB Journal, 10: $979-985$.

Rountree, M.R., Bachmann, K.E., Baylin, J.G. 2001. DNA methylation chromatin inheritance and cancer. Oncogene, 20: 3156-3165.

Schneider, R.B., Bannister, A.J., Myers, F.A., Thorne, A.W., Crane-Robinson, C., Kouzarides, T. 2004. Histone H3 lysine 4 methylation patterns in higher eukaryotic genes. Nature Cell Biology, 6: 73-78.

Shenkin, A. 2004. Basics in clinical nutrition: Trace elements and vitamins in parenteral and enteral nutrition. eSPEN, the European e-Journal of Clinical Nutrition and Metabolism, 3: e293-297.

Sie, K.K., Medline, A., vanWeel, J., Sohn, K.J., Choi, S.W., Croxford, R., Kim, Y.I. 2011. Effect of maternal and post weaning folic acid supplementation on colorectal cancer risk in offspring. Gut, 60: $1687-1694$.

Stover, F. 2008. Folate-mediated one-carbon metabolism. Vitamins and Hormones, 79: 1-44.

Stover, P.J. 2011. Polymorphisms in 1-Carbon, meta- 
bolism, epigenetics and folate-related pathologies. Journal of Nutrigenetics and Nutrigenomics, 4: 293-305.

Stover, P.J. 2007. Humn nutrition and genetic variation. Food and Nutrition Bulletin, 28: 101-115.

Stover, P.J. 2004. Physiology of folate and vitamin B12 in helth and disease. Nutrition Reviews, 64: 212.

Surani, J.A. 2013. DNA methylation dynamics during the mammalian life cycle. Philosophical Transactions of Royal Society of London Biological Sciences, 368.

Taylor, C.G. 2005. Zinc, the pancreas and diabetes: Insights from rodent studies and future directions. Biometallics, 18: 305-312.

Van Mierlo, L.A. 2006. Blood pressure response to calcium supplementation;a meta-analysis of randomized controlled trials. Journal of Human Hypertension, 20: 571-580.

Wade, P.A. 2001. Methyl CpG binding proteins and transcriptional repression. Bioessays, 23: 11311137.

Waddington, C.H. 1953. Epigenetics and evolution. Proceedings of the Society of Experimental Biology, 7: 186-199.

Wakefield, L., Boukouvala, S., Sim, E. 2010. Characteriztion of $\mathrm{CpG}$ methyltion in the upstream control region of mouse Nat 2: Evidence for a gene environment interaction in the polymorphic gene implicated in folate metabolism. Gene, 452: 16-21.

Waterland, R., Garza, C. 1999. Potential mechanisms of metabolic imprinting that lead to chronic disease. American Journal of Clinical Nutrition, 69: 179197.

Winter-Vann, A.M., Kanen, B.A., Bergo, M.O., Young, S.G., Melnyk, S., James, S.J., Casey, P.J. 2003. Targeting Ras signaling through inhibition of carboxyl methylation: An unexpected property of methotrexate. Proceedings of National Academy of Sciences USA, 100: 6529-6534. 